\title{
Partial Nephrectomy
}

National Cancer Institute

\section{Source}

National Cancer Institute. Partial Nephrectomy. NCI Thesaurus. Code C51928.

The surgical removal of a portion of a kidney. 